\title{
A REVIEW OF MOBILE AD-HOC NETWORKS REACTIVE OR ON-DEMAND ROUTING PROTOCOLS
}

\author{
B.Yuvanithya \\ Research Scholar \\ Department of PG \& Research in Computer Science \\ Dr.N.G.P Arts and Science College (Autonomous) \\ Coimbatore-48, India.
}

\author{
V.S.Jagadeeswaran \\ Research Supervisor, Assistant Professor \\ Department of PG \& Research in Computer Science \\ Dr.N.G.P Arts and Science College (Autonomous) \\ Coimbatore-48, India.
}

\begin{abstract}
A MANET (Mobile Ad-hoc network) is a self-configuring infrastructure less network of portable routes associated by wireless connections. All mobile nodes play the role of a router moreover as a host. The topology of MANET is not stable. It is similar to the TCP/IP stack with little modification for end to end transmission. Routing is the challenging task in MANET because nodes moves freely and works as a host and router at the same time in different environment. Many researchers have proposed many routing protocols which work well in different conditions. This paper provides a survey of different reactive routing protocol in MANET.
\end{abstract}

Keywords : MANET; Reactive; Proactive; Hybrid; AODV; DSR; ODMRP; MAODV.

\section{INTRODUCTION}

Wireless network is an interconnection of one or more systems which provides service to mobile users within a particular region. Wireless networks are configured into two main types one is infrastructure or single hop wireless network, where access points are used for the communication of mobile nodes and the other is the Ad hoc network in which mobile nodes acts both as a router and a host. The nodes in the network communicate with other node via multi-hop links. Base station is fixed to provide communication in an infrastructure network, whereas in Ad hoc network mobile nodes are self organized and configured for sending and receiving data.

MANETs are a group of self-determining mobile nodes. Mobile nodes moving arbitrarily in a network thus no permanent topology is maintained [9]. Due to this various protocols have been proposed for Mobile ad hoc network (MANET).The the characteristics of MANET are

- Distributed operation

- Multi hop routing

- Autonomous terminal

- Dynamic topology

- $\quad$ Light weight terminals

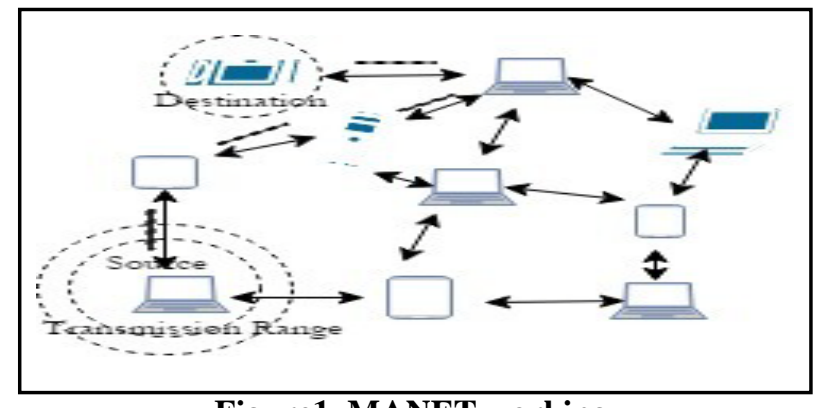

Figure1. MANET working

\section{ROUTING PROTOCOLS}

Routing protocols are responsible in finding the best shortest path from sender to the destination for sending packets [5]. Ad hoc routing protocol is used by a mobile node which acts as a host and router to route packets to its destination [4]. MANET protocol stack and TCP/IP model is quiet similar.

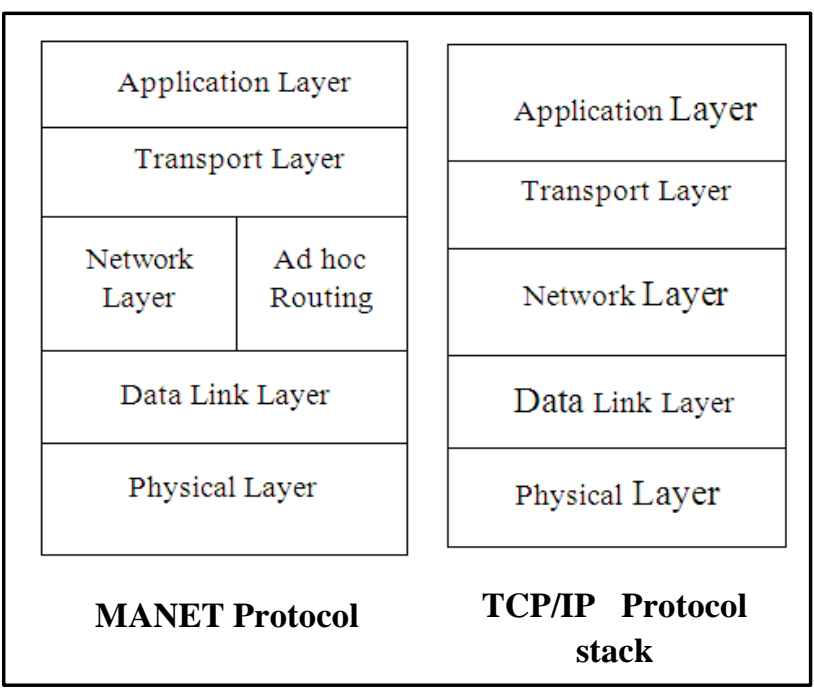

Figure2. MANET Protocol stack

The forwarding is done with the help of routing table. The table keeps the information about target or recipient address; sequence number, next hop address in addition to hop count. MANET routing protocols can be classified in various ways. Classification of routing protocols as table driven and source initiated depending upon the strategy of routing while based on structure of network the routing protocols are classified as flat routing (both table driven and source initiated), hierarchical routing and geographic assisted position routing [11], [2], [20]. 
The classification is as:

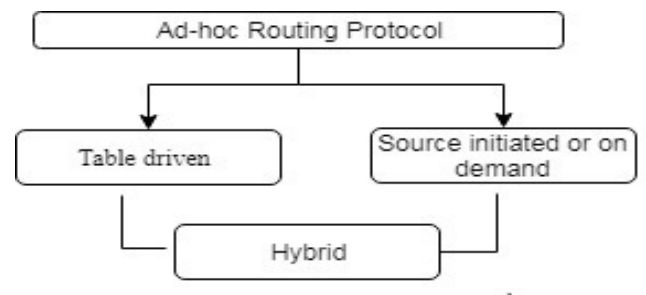

Figure3.classification of Ad-hoc routing protocol

\section{A. Table - Driven Protocol}

The nodes keep up the information of fresh routes of all the nodes and also its routes in its routing table [12]. To refresh the routes flooding mechanism is utilized [7]. The source node transmits the packet if a route exists in routing table, thus transmission occurs without delay. If a route is not found, then the packets should wait in the queue until the routing information is received by the node.

The smaller network uses table driven protocol, because the routing table is updated every time which leads to routing overhead [18]. The benefit of proactive protocol is that the session begins immediately as routes are accessible when required. The drawback is the control overhead in larger network or a network with moving nodes and control traffic. For instance; DSDV, WRP, FSR, OLSR and so forth.

\section{B. On Demand or Reactive Protocol}

In this sort of routing protocol, the route is setup on request when two nodes need to convey. The two primary elements of this sort of protocol are route discovery as well as route maintenance. Route detection is performed to find the path to arrive at the target [15], [16]. The routing table discards the routing information once the connection is terminated. The benefit of this sort of protocol is the decrease in routing overhead as compared to proactive protocol. When a route is needed from source to destination it takes a finite latency for the route information, which is the main disadvantage of reactive routing protocol. For instance; AODV, DSR, TORA and so forth.

\section{Hybrid Routing Protocol}

It is the permutation of source initiated or table driven. Hybrid routing protocol attempts to reduce the traffic overhead in proactive systems and routing table is maintained for reducing the time for route discovery in reactive systems [4], [13], [23]. For instance; ZRP, ZHLS, and so forth [23].

\section{AD-HOC REACTIVE ROUTING PROTOCOL}

\section{A. Ad hoc On-demand Distance Vector Protocol}

This is a reactive routing protocol. AODV does not maintain the routes but it discovers the route quickly when it is required [3], [21]. The nodes are communicated by dropping the control overhead. If the destination has related routes the node choose the path which have highest sequence number.

The communication by using four sorts of messages

i. Route Request, ii. Route Reply,

iii. Route Error,

iv. HELLO messages

Route discovery is done with RREQ and RREP whereas route maintenance is carried with RERR and HELLO messages [11], [12]. The source node broadcasts a RREQ when it needs to transmit a data to a destination which is unknown. When an intermediate node gets a RREQ-Route Request it creates a route from this to the source node. Once the recipient node initially makes conformation of RREQ and then Route Reply is sent. The sender rebroadcasts the Route Request (RREQ) once the TTL gets expired. The transmission is initiated from the source node in turn of receiving the RREP which is sent by the receiving node in its turnaround path [22].

The connections are monitored by utilizing HELLO messages. There will be a link breakage if these messages are not received properly. The transmission is terminated if the source node moves during an active session. The host creates Route error when it moves in the path. Route error message is sent to predecessor nodes. The DSR data packet header contains the entire route information which leads to bandwidth wastage and the performance is reduced. The routing data is kept up all nodes thus the performance is enhanced. Forwarding tables have been utilized by AODV on the other hand DSR utilizes source routing [10].

\section{B. DYNAMIC SOURCE ROUTING :}

This comes below reactive protocol. It is utilized in wireless ad hoc networks. It finds the route only when it is needed. Dynamic source routing allows the system to be self-organizing with self-configuring without an existing infrastructure [8].

The two primary elements of this sort of protocol are route discovery as well as route maintenance. [1], [13]. RREQ and RREP are utilized for route discovery. The source routing algorithm is used by DSR where every node contains the full routing information and the caching technology is used by every node to maintain the routing information. The packets are forwarded commencing the source node to its recipient node, merely when the route cache has the information of routing [19]. Incase if no routes are available the Route request is broadcasted. The intermediate node on receiving the RREQ packet checks for valid route in the route cache, if route is not found then the node adds its address to the route record and forwards the packet. The recipient generates the Request Reply despite the fact that RREQ arrived. Path breaks are managed by route maintenance. RERR is transmitted once the route information is deleted from the route cache. The transmission complexity takes place in the data link layer. Every node recieves error packet. The control packet is sent over the broken link.

\section{On-Demand Multicast Routing Protocol}

This comes under reactive protocol. ODMRP utilizes a forwarding group concept. Soft-state method is utilized to uphold multicast group [1], [6]. The member does not entail several control messages to depart the group. When a multicast source has no route to send the packets it broadcasts the Join-Query control packet and sends to the entire network. 
The intermediate node on in receipt of Join-Query packet, it keep track of the sequence number along with the source ID inside the message cache to evade repetition.The multicast receiver subsequently getting the Join-Query packet; generates, broadcasts a "Join Reply". The node which receives a Join Reply checks its own ID with the next hop node ID, if it is matched then it sets Forwarding Group Flag. By extracting the information as of the routing table the next hop ID be occupied.

The source or query issuing node can be left from the group exclusive of any control message but simply without transmitting the Join-Query packet. As well the recipient can be left from the group by not sending back Join Reply packets.

\section{MAODV - Multicast Ad-hoc On-demand Distance Vector Protocol}

This is a sort of reactive protocol that finds a route on request. The development from AODV is MAODV. The node creates a Route Request as soon as it desires to send the information otherwise to bond the multicast group [14]. The persons in that multicast group can react to RREQ. The RREQ accredited by an intermediary node rebroadcasts the RREQ if that host is not a member or when it does not have a route [17].

The node which responds updates the next hop of requesting node in its routing table, and RREP is sent (unicast) back to source node. The routing or transmission path is established after route reply is received. Several responses have been received as the result of transmitting route request in group of destinations. The shortest hop count and the greatest sequence number are kept for a particular timeframe in the source node. The MACT is sent to the next hop.

The foremost member is the person in charge of that group and is answerable for maintaining as well as broadcasting the multicast group sequence number [3]. This is done with the help of Group Hello message; consists of extensions (comprises of multicast group ID address and sequence number of that multicast group leader). The head of the group maintains the broken links.

\section{CONCLUSION}

Mobile Ad hoc Network is a challenging feature where routing protocols is an important component for communication. Because node has the aspect of moving freely within the environment or network thus it has no stable topology. The reactive protocols are used on demand and some protocols are discussed in this paper. This protocol creates routes only on demand hence routing table is created when node request packets from source to destination and it exists until the connection exists. Each protocols works well in some cases and also has certain drawbacks.

\section{V.REFERENCES}

[1] Gupta, Surabhi, and Manish Saxena. "Comparative Analysis of QoS Routing Protocols in MANETS: Unicast \& Multicast." International Journal of Engineering Science 6930 (2016).
[2] Nazir, Muhammad Kashif, Rameez U. Rehman, and Atif Nazir. "A Novel Review on Security and Routing Protocols in MANET." Communications and Network 8.04 (2016): 205.

[3] Rajani, K. C., P. Aishwarya, and S. R. Meenakshi. "A review on multicasting routing protocols for mobile ad-hoc wireless networks." Communication and Signal Processing (ICCSP), 2016 International Conference on. IEEE, 2016.

[4] Mukhija, Arun, and R. Bose. "Reactive Routing Protocol for Mobile Ad-hoc Networks." Department of Mathematics Indian Institute of Technology (2001).

[5] Gorantala, Krishna. "Routing protocols in mobile adhoc networks." A Master’thesis in computer science, pp-1-36(2006).

[6] Lee, Sung-Ju, Mario Gerla, and Ching-Chuan Chiang. "On-demand multicast routing protocol." Wireless Communications and Networking Conference, 1999. WCNC. 1999 IEEE. Vol. 3. IEEE, 1999.

[7] Lois, Keren. "Comparative Study of Reactive Routing Protocols for MANETs."

[8] Johnson, David B., David A. Maltz, and Josh Broch. "DSR: The dynamic source routing protocol for multi-hop wireless ad hoc networks." Ad hoc networking 5 (2001): 139-172.

[9] Patel, Daxesh N., et al. "A survey of reactive routing protocols in MANET." Information Communication and Embedded Systems (ICICES), 2014 International Conference on. IEEE, 2014.

[10] http://webhome.csc.uvic.ca/ wkui/Courses/wireless/L ecture4.pdf

[11] Srivastava, Ashish, et al. "Survey and overview of Mobile Ad-Hoc Network routing protocols." Advances in Engineering and Technology Research (ICAETR), 2014 International Conference on. IEEE, 2014.

[12] Raut, Surendra H., and Hemant P. Ambulgekar. "Proactive and Reactive Routing Protocols in Multihop Mobile Ad hoc Network." International Journal of Advanced Research in Computer Science and Software Engineering 3.4 (2013).

[13] Tanenbaum, Andrew S. "Computer networks, 4-th edition." ed: Prentice Hall (2003).

[14] Kunz, Thomas, and Ed Cheng. "Multicasting in adhoc networks: Comparing MAODV and ODMRP." Proceedings of the Workshop on Ad hoc Communications. Bonn, Germany, 2001.

[15] Rodríguez-Covili, J., Ochoa, S. F., \& Pino, J. A. (2012). High level MANET protocol: Enhancing the communication support for mobile collaborative work.Journal of Network and Computer Applications, 35(1), 145-155.

[16] Mohammed Aziz Ahmed, Mohammed Sirajuddin, Nazia Kouser "A Review on Reactive \& Proactive routing Protocols and Security Breaches and Remedies in MANET's” (IJCSIT)International Journal of Computer Science and Information Technologies, Vol.7 (4), 2016, 1962-1965.

[17] Royer, Elizabeth M., and Charles E. Perkins. "Multicast operation of the ad-hoc on-demand distance vector routing protocol." Proceedings of the 
5th annual ACM/IEEE international conference on Mobile computing and networking. ACM, 1999.

[18] Lesiuk, B. Cameron. "Routing in ad hoc networks of mobile hosts." Mech590 Report, University of Victoria, British Columbia (1998).

[19] Johnson, David, Yin-chun Hu, and David Maltz. The dynamic source routing protocol (DSR) for mobile ad hoc networks for IPv4. No. RFC 4728. 2007.

[20] Rupérez Cañas, Delfín, et al. "A Family of ACO Routing Protocols for Mobile Ad Hoc Networks." Sensors 17.5 (2017): 1179.
[21] http://cdn.intechopen.com/pdfs/12861.pdf

[22] Anand, Anjali, and Himanshu Aggarwal. "Dynamic source routing-an ondemand routig protocol in mobile adhoc networks." Journal of Global Research in Computer Science4.5 (2013): 13-16.

[23] Jawandhiya PM, Mangrulkar RS, Atique M, Ali MS. A Novel Hybrid Routing Protocol for Mobile Adhoc Network. Int. J. Advancements in Technology. 2010 Oct;1(2):185-96. 